OPEN ACCESS

Edited by:

Dawit Kidane

University of Texas at Austin,

United States

Reviewed by:

Zehua Bian,

Affiliated Hospital of Jiangnan

University, China

Lu Zhang,

Henan University, China

${ }^{*}$ Correspondence: Zifang Song

zsong@hust.edu.cn

tThese authors have contributed equally to this work

Specialty section:

This article was submitted to Gastrointestinal Cancers,

a section of the journal

Frontiers in Oncology

Received: 26 November 2020 Accepted: 09 February 2021 Published: 22 March 2021

Citation:

Du X, Zhang C, Yin C, Wang W, Yan X, Xie D, Zheng X, Zheng Q, Li M and Song Z (2021) High BLM Expression Predicts Poor Clinical Outcome and Contributes to Malignant Progression in Human Cholangiocarcinoma.

Front. Oncol. 11:633899. doi: 10.3389/fonc.2021.633899

\section{High BLM Expression Predicts Poor Clinical Outcome and Contributes to Malignant Progression in Human Cholangiocarcinoma}

\author{
Xiaolong Du ${ }^{1 \dagger}$, Chen Zhang ${ }^{1 \dagger}$, Chuanzheng Yin ${ }^{1}$, Wenjie Wang ${ }^{1}$, Xueke Yan ${ }^{1}$, Dawei Xie ${ }^{2}$, \\ Xichuan Zheng ${ }^{1}$, Qichang Zheng ${ }^{1}$, Min $\mathrm{Li}^{1}$ and Zifang Song ${ }^{1 *}$ \\ 1 Department of Hepatobiliary Surgery, Union Hospital, Tongji Medical College, Huazhong University of Science and \\ Technology, Wuhan, China, ${ }^{2}$ State Key Laboratory of Molecular Oncology, National Cancer Center/National Clinical Research \\ Center for Cancer/Cancer Hospital, Chinese Academy of Medical Sciences and Peking Union Medical College, Beijing, China
}

Molecular mechanisms underlying the tumorigenesis of a highly malignant cancer, cholangiocarcinoma (CCA), are still obscure. In our study, the CCA expression profile data were acquired from The Cancer Genome Atlas (TCGA) database, and differentially expressed genes (DEGs) in the TCGA-Cholangiocarcinoma (TCGA-CHOL) data set were utilized to construct a co-expression network via weighted gene co-expression network analysis (WGCNA). The blue gene module associated with the histopathologic grade of CCA was screened. Then, five candidate hub genes were screened by combining the coexpression network with protein-protein interaction (PPI) network. After progression and survival analyses, bloom syndrome helicase (BLM) was ultimately identified as a real hub gene. Moreover, the receiver operating characteristic (ROC) curve analysis suggested that BLM had a favorable diagnostic and predictive recurrence value for CCA. The gene set enrichment analysis (GSEA) results for a single hub gene revealed the importance of cell cycle-related pathways in the CCA progression and prognosis. Furthermore, we detected the BLM expression in vitro, and the results demonstrated that the expression level of BLM was much higher in the CCA tissues and cells relative to adjacent non-tumor samples and normal bile duct epithelial cells. Additionally, after further silencing the BLM expression by small interfering RNA (siRNA), the proliferation and migration ability of CCA cells were all inhibited, and the cell cycle was arrested. Altogether, a real hub gene (BLM) and cell cycle-related pathways were identified in the present study, and the gene BLM may be involved in the CCA progression and could act as a reliable biomarker for potential diagnosis and prognostic evaluation.

Keywords: cholangiocarcinoma, prognosis, progression, co-expression network analysis, bioinformatics

\section{INTRODUCTION}

Cholangiocarcinoma (CCA) is one of the most frequent primary biliary duct malignancies and accounts for $3 \%$ of all gastrointestinal neoplasias $(1,2)$. Since CCA possesses the characteristics of high malignancy, insidious onset, and rapid progression, the prognosis of patients with CCA is often poor $(3,4)$. Large-scale clinical research revealed that the 5 -year survival rates were 5 and $17 \%$ 
for intrahepatic CCA (iCCA) and extrahepatic CCA (eCCA), respectively, for the cases diagnosed between 2000 and 2007 in Europe (5). In the USA alone, CCA accounts for approximately 5,000 deaths per year (6). Moreover, since its early symptoms are not obvious and CCA lacks a highly efficient disease screening biomarker, many patients have frequently lost the chance of radical surgery upon the first diagnosis (7). Furthermore, as the pathogenesis of CCA is rather complicated, there is still no satisfying targeted treatment option available for CCA in the age of individualized medicine (8). Therefore, there is an urgent need to explore the molecular pathogenesis of CCA and reveal the biomarkers closely related to the diagnosis, occurrence, progression, and prognosis of CCA.

Due to advances in sequencing and microarray technologies, bioinformatics has begun to play an increasingly important role in various fields of life sciences $(9,10)$. The development of largescale "omics" research methods such as genomics, proteomics, and transcriptomics has brought about a vast amount of biological information data (11). Gene co-expression networks can describe and cluster multiple genes sharing high-expression correlations in microarray data or high-throughput sequencing data and ultimately be used to establish key regulatory genes (12). A weighted gene co-expression network analysis (WGCNA), as one of the most representative analytical methods of a gene network, has provided significant biological information for the research on multiple species such as humans, mice, and yeast $(13,14)$. In this study, a WGCNA network was constructed, and genes with similar expression patterns were incorporated into the identical modules. By relating the results of the module to the corresponding clinical data, the modules that were mostly associated with the CCA progression were found. Finally, after a range of screening and validation tests, we identified a bloom syndrome helicase (BLM) gene that could indeed promote the tumor progression and predict the prognosis of CCA.

\section{MATERIALS AND METHODS}

\section{Data Sets and Study Design}

RNA-sequencing (RNA-seq) data (Illumina RNA Seq V2, Illumina, San Diego, CA) and the corresponding clinical data on CCA samples were downloaded from The Cancer Genome Atlas (TCGA) database (http://cancergenome.nih.gov/). This CHOL data set included 36 CCA samples and 9 corresponding adjacent non-cancerous samples, and its clinical data were composed of tumor histologic grade, pathological stage, and numerous follow-up information. The messenger RNA (mRNA) expression profile and clinical data were employed to search for differentially expressed genes (DEGs) and construct co-expression networks. The microarray data sets GSE76297 and GSE132305 were acquired from the gene expression omnibus (GEO) database of the National Center for Biotechnology Information (NCBI) database (https://www.ncbi.nlm.nih.gov/). These two data sets were generated on the platforms of the Affymetrix Human Transcriptome Array 2.0 and Affymetrix Human Genome U219 Array, respectively. The data set GSE76297 contained 91 iCCA tissue and 92 non-neoplastic samples, and the data set GSE132305 included 182 eCCA samples and 38 corresponding adjacent non-cancerous samples. These two data sets were used to further validate our candidate hub genes.

\section{Differentially Expressed Gene Screening and Principal Component Analysis}

The "TCGAbiolinks" R package (15) was used to identify the DEGs between adjacent normal tissues and CCA samples in the CHOL data set. Significance analysis with a false discovery rate $(\mathrm{FDR})<0.01$ and $\mid \log _{2}$ fold change $(\mathrm{FC}) \mid \geq 1$ was adopted to choose the genes for a network construction. To explore gene expression patterns between the CCA samples and adjacent tissues, the top 500 DEGs were included in the principal component analysis (PCA) utilizing the R package "pca3d" (https://cran.r-project.org/web/packages/pca3d/). The first three principal components were utilized and plotted to show the expression pattern of the two groups.

\section{Weighted Gene Co-expression Network Analysis}

R package of "WGCNA" (16) was utilized to build a co-expression network for the qualified expression data profiles of filtered DEGs with the available clinical data. First, we retained the RNA-seq data of the filtered CCA tissues if they proved to be good samples. Second, outlier samples were culled after a sample clustering via correlation analysis. Third, based on a Pearson correlation analysis, a matrix of similarity for all pairs of kept genes was constructed. Then, appropriate soft-thresholding was chosen to achieve a scale-free co-expression network. Next, the adjacency matrix was converted to a topological overlap matrix (TOM). In line with the TOM-based dissimilarity method, genes were assigned to the different modules. Here, we set the softthresholding power to 9 (scale-free $R^{2}=0.913$ ), the cut height to 0.25 , and the minimal module size to 30 to identify key modules. The module most relevant to clinical traits was chosen to screen co-expression hub genes.

\section{Function Enrichment Analyses}

We performed gene ontology (GO) and Kyoto Encyclopedia of Genes and Genomes (KEGG) enrichment analyses utilizing the R package "clusterprofiler" (17). GO terms or KEGG pathways with $p<0.05$ were regarded as statistically significant and visualized by R package “GOplot” (18).

\section{Candidate Hub Genes Identification}

In this research, a module of interest was determined, and coexpression hub genes were specified as high module connectivity (module membership $>0.8$ ) and high clinical trait significance (gene significance $>0.4$ ). Moreover, the gene list of this key module was submitted to the STRING database to build a protein-protein interaction (PPI) network (19), which was then visualized by Cytoscape software (20). PPI network hub genes were defined as the top 20 nodes ranked by degree using the plugin cytoHubba. Hub genes shared by the 2 networks were selected as the candidates for further validation and analysis. 


\section{Validation and Survival Analysis of Candidate Hub Genes}

To screen real hub genes, we used R package "ggstatsplot" to explore the candidate gene expression pattern between CCA and adjacent normal tissue in the TCGA-CHOL data set, GSE76297, and GSE132305; the candidate genes that were correlated with a clinical feature (histological grade) were analyzed by using the tumor samples from the TCGACHOL data set. Survival analysis was also performed for the candidate genes in gene expression profiling interactive analysis (GEPIA), an online database (http://gepia.cancer-pku. cn/index.html), and validated in the data set GSE107943 by using online web server OSchol (http://bioinfo.henu.edu. cn/CHOL/CHO-L_GSE107943.jsp). Independent sample $t$-tests were used as appropriate, and $p<0.05$ was considered as statistically significant.

\section{Methylation Analyses and Efficacy Evaluation of Real Hub Genes}

An online tool DiseaseMeth 2.0 (21) contains a mass of methylome data and annotations on the DNA methylation status in multiple diseases. We exploited this web tool to compare the methylation level of the real hub gene between the CCA and adjacent normal tissues. Additionally, the association between the BLM expression and DNA methylation status was explored via the web tool MEXPRESS (22), which can integrate and visualize gene expression, DNA methylation, and clinical information from the TCGA database. The receiver operating characteristic (ROC) curves were plotted, and the area under the curve (AUC) was calculated with the "pROC" R package (23) to assess the capability of BLM gene of distinguishing tumor and normal samples as well as recurrent and non-recurrent CCA.

\section{Gene Set Enrichment Analysis}

Based on the median expression value of the real hub gene, CCA samples of the TCGA-CHOL data set were divided into low- or high-expression groups. To explore potential functions of the real hub gene, gene set enrichment analysis (GSEA) was performed to detect which KEGG pathways were enriched (24). Terms with FDR $<0.05$ were visualized by R package "ggplot2" (https:// cloud.r-project.org/web/packages/ggplot2/).

\section{Cell Culture}

Human CCA cell lines (QBC939 and RBE) and human biliary epithelial cell line (HiBEC) were obtained from Sciencell (Carlsbad, USA) and the Cell Bank of the Chinese Academy of Sciences (Shanghai, China) and maintained in Roswell Park Memorial Institute (RPMI) 1,640 medium (Gibco, NY, USA) containing 10\% fetal bovine serum (FBS) (ScienCell, San Diego, CA, USA) and $100 \mathrm{U} / \mathrm{ml}$ penicillin-streptomycin (Beyotime, Shanghai, China) at $37^{\circ} \mathrm{C}$ in a humidified incubator supplied with $5 \% \mathrm{CO} 2$.

\section{Transfection}

Small interfering RNA (SiRNA) silencing was attained by transient transfection with Lipo3000 (Invitrogen, Waltham, MA, USA). BLM siRNAs (si-RNA1 and si-RNA2) were purchased from RiboBio (RiboBio, Guangzhou, China), with scramble RNA serving as a negative control (scramble). The sequences for siRNA1 and si-RNA2 were 5'-GGATGTTCTTAGCACATCA-3' and GACTCAGAATGGTTAAGCA, respectively. The scramble sequence was $5^{\prime}$ - TTCTCCGAACGTGTCACGTdTdT-3'. The siRNA knockdown efficiency was examined by qRT-PCR and western blotting assays.

\section{CCK8 Assay}

Cells transfected with si-BLM or scramble were seeded into 96well-plates at $4 \times 10^{3}$ cells per well and cultured for 0-3 days. Then, $10 \mu \mathrm{l}$ of CCK8 solution (Dojindo, Kumamoto, Japan) was added to the fresh culture medium, and the cells were incubated at $37^{\circ} \mathrm{C}$ for $1.5 \mathrm{~h}$. Afterward, the absorbance at $450 \mathrm{~nm}$ was determined by using a microplate reader.

\section{Wound-Healing Assay}

For the wound-healing assay, transfected cells were inoculated into 6-well-plates. After the cell attachment, a sterile plastic microtube head was used to generate scratch wounds. Then, the cells were washed twice with PBS, and initial wounds were recorded by a microscope. After incubation for $36 \mathrm{~h}$ at $37^{\circ} \mathrm{C}$, the current wound space was captured. The quantity of wound closure was defined as the mean percentage of the distance of cell migration compared with the initial wound width.

\section{Transwell Assay}

Cell migration ability was further evaluated via transwell assays. Briefly, transfected cells $\left(3 \times 10^{4}\right)$ were plated into $8.0-\mu \mathrm{m}$ pore polycarbonate membrane chambers (Corning, NY, USA) with $200 \mu \mathrm{l}$ of serum-free medium, and then the chambers were placed in 24-well-plates containing $600 \mu \mathrm{l}$ of complete medium. After $24 \mathrm{~h}$, the cells migrating to the lower surface were fixed with a formaldehyde solution and stained with $0.1 \%$ crystal violet solution. The number of migrated cells was counted in five randomly selected visual fields of each membrane with a microscope.

\section{Quantitative Real-Time PCR Analysis}

Total RNA from the cultured cells was extracted by using TRIzol (Invitrogen, Carlsbad, CA, USA), and then it was reverse transcribed into cDNA via PrimeScript RT Master Mix (Takara, Dalian, China). Subsequently, quantitative real-time PCR (RTqPCR) was conducted by utilizing the TAKARA PCR Kit, run on a StepOnePlus Real-Time PCR system (Applied Biosystems, CA, USA). $\beta$-actin was chosen as an internal reference. The relative expression abundance of genes was assessed by using the $2^{-\Delta \Delta \mathrm{CT}}$ approach (25). Primers were provided as follows: $\beta$-actin, forward $5^{\prime}$-TGCTATGTTGCCCTAGACTTCG- $3^{\prime}$ and reverse 5'-GTTGGCATAGAGGTCTTT ACGG-3'; and BLM, forward 5' - CAGACTCCGAAGGAAGTTGTATG' and reverse 5' - TTTGGGGTGGTGTAAC AAATGAT-3'.

\section{Western Blot Analysis}

For the total protein extraction, the treated cells were lysed by RIPA buffer (Beyotime, Shanghai, China) with protease inhibitors (Servicebio, Wuhan, China). Then, the same amount of whole-cell lysates was loaded on the sodium dodecyl 
sulfate-polyacrylamide gel electrophoresis (SDS-PAGE) and then transferred onto PVDF membranes. After $1 \mathrm{~h}$ of blocking, proteins were incubated with anti- $\beta$-actin antibodies (abs132001, Absin, Shanghai, China), anti-GAPDH antibodies (abs132004, Absin, Shanghai, China), and anti-BLM antibodies (abs122169, Absin, Shanghai, China) and subsequently with appropriate secondary antibodies (Servicebio Technology, Wuhan, China). Protein bands were detected and quantified via ImageLab (BioRad, Hercules, CA, USA).

\section{Immunohistochemistry Staining}

A tissue microarray (TMA) containing 27 CCA specimens and 9 paracancerous samples was procured from Shanghai Outdo Biotech (Shanghai, China). In short, paraffin sections were first deparaffinized, antigen retrieval was conducted in citrate buffer ( $\mathrm{pH}$ 6.0), and endogenous peroxidase activity was blocked in $0.3 \% \mathrm{H}_{2} \mathrm{O}_{2}$. The slides were continuously incubated with the indicated primary and secondary antibodies until visualization with peroxidase and 3,30-diaminobenzidine tetrahydrochloride. Immunohistochemical staining was scored semiquantitatively according to the percentage and intensity of positively stained cells as follows: 0 : $<5 \%$ positive cells; 1 : from 5 to $24 \%$ positive cells; 2: from 25 to $49 \%$ positive cells; 3: from 50 to $74 \%$ positive cells; and 4 : more than $75 \%$ positive cells. The intensity was scored as 0 for the absence of staining, 1 for weak, 2 for moderate, and 3 for strong staining. Staining index score $=$ intensity $\times$ positive rate (absent, $0-1$; mild, 24 ; moderate, 5-8; and strong, 12). A staining index score $\geq 8$ represented a high expression, while scores $<8$ represented a low expression. Immunohistochemical slices were observed by utilizing an Olympus BX microscope.

\section{Flow Cytometry}

The treated cells were washed with pre-cooled PBS twice and fixed in $70 \%$ pre-cooled ethanol overnight at $4^{\circ} \mathrm{C}$ and washed again with PBS twice. The cells were resuspended in $1 \mathrm{ml}$ $\mathrm{PI} /$ Triton X-100 staining solution containing $0.2 \mathrm{mg}$ RNase A for $15 \mathrm{~min}$ at $37^{\circ} \mathrm{C}$ and then analyzed via flow cytometry. Cells were assayed at each cell cycle.

\section{Statistical Analysis}

Each cell experiment was carried out thrice. All measurement data were presented as the mean \pm SEM. Statistical data analysis was conducted by a one-way ANOVA or unpaired two-tailed Student's $t$-test, as appropriate. Values of $p<0.05$ were deemed to indicate statistical significance.

\section{RESULTS}

\section{Screening of DEGs}

After data preprocessing, data normalization, data filtering, and quality assessment by "TCGAbiolinks", 6,219 DEGs were identified (4,087 upregulated and 2,132 downregulated) with the following threshold: FDR $<0.01$ and $|\log F C| \geq 1$. Then, the screened genes were utilized for a follow-up analysis. The volcano plot for all DEGs is displayed in Figure 1A; the PCA of the top 500 DEGs is shown in Figure 1B.

\section{WGCNA and Key Module Identification}

The "WGCNA" R package was utilized to build a co-expression network, and DEGs with similar expression relationships were assigned into the same modules by average linkage clustering. Here, we selected the power of $\beta=9$ (scale-free $R^{2}=0.913$ ) to assure a scale-free network (Figures 2A,B), then 29 modules were determined (Figure 2C) in the subsequent experiments. The module that was most significantly correlated with a tumor grade indicated a great value in predicting the CCA progression, and the ME of the blue module indicated its high correlation with the CCA progression (Figure 2D). Moreover, we also found that the module significance (MS) of the blue module was the highest among all the modules that were positively correlated with disease progression (Figure 2E). Afterward, GO and KEGG analyses were performed to uncover the potential role of the members within the blue module. The most significant enrichment results are illustrated in Figures 3A-D. The biological function analysis suggested that blue module genes mainly participated in the cell cycle regulation and pathways in multiple cancers.

\section{Identification of Hub Genes}

According to the scheme mentioned above, a total of 94 genes highly connected to the key module were selected as coexpression network hub genes in the blue module (Figure 4A). Additionally, we also built a PPI network for all blue module genes, which was composed of 123 nodes and 271 edges (Figure 4B). Then, using the cytoHubba plugin, the top 20 nodes associated with over 8 nodes were identified as hub genes in the PPI network (Figure 4C). Eventually, five common network hub genes-BLM, GGH, RPS3, NUP107, and CCT2were identified as potential candidates for further validation and analysis (Figure 4D).

\section{Validation of Real Hub Gene}

To verify the diagnostic value of the candidate genes, first, we examined the expression relationship of the candidate genes between CCA and normal controls using the TCGA$\mathrm{CHOL}$ data set. It showed that the expression of candidate genes was upregulated in the CCA sample, except for the GGH gene (Figure 5A). Then, among the CCA samples in the TCGA-CHOL data set, we explored their correlations with the pathological grade of clinical features. The results suggested that four candidate genes were significantly higher in the lowdifferentiation tumor samples, indicating a role in the CCA progression (Figure 5B). To further examine the prognostic value of candidate genes in CCA, we conducted a subgroup (high- and low-expression group) survival analysis of these 5 genes in the TCGA-CHOL data set, and their prognostic value was validated in the data set GSE107943 by using the online tool OSchol (26). Kaplan-Meier (KM) survival curves indicated that only higher BLM expression was correlated significantly with worse disease-free survival (Figures 5C,D). Furthermore, the expression pattern of BLM in both the eCCA data set GSE132305 and the iCCA data set GSE76297 suggested that the expression of BLM in tumor tissue was enhanced compared with paracancerous samples (Figures 6A,B). ROC curves were then 

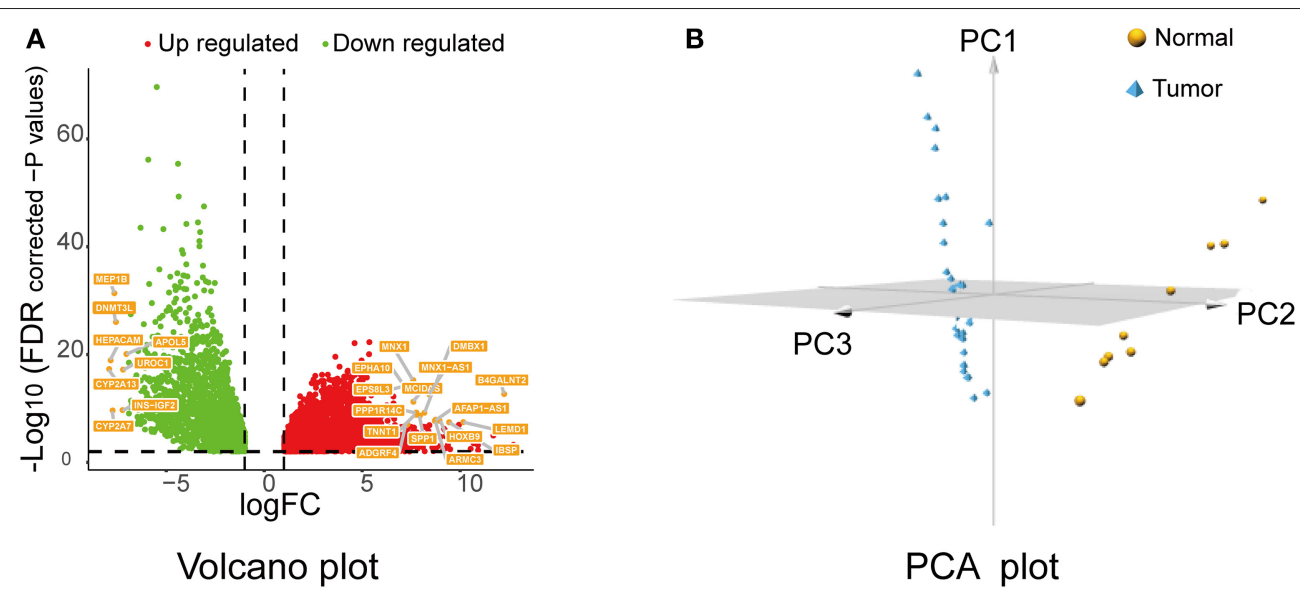

FIGURE 1 | Volcano plot and PCA of DEGs. (A) Volcano plot of the DEGs between cholangiocarcinoma (CCA) tissues and normal controls of The Cancer Genome Atlas (TCGA) CHOL data set. Red means upregulated DEGs, and green indicates downregulated DEGs. (B) PCA plot for the TCGA-CHOL data set based on top 500 DEGs. PCA, principal component analysis; DEGs, differentially expressed genes.

generated to assess the capacity of BLM to differentiate recurrent and non-recurrent CCA as well as tumor and non-tumor tissues (Figures 6C,D). The results above identified BLM as the real hub gene most closely related to the progression of CCA and as having an essential value in potential diagnosis and prognosis assessment of the disease.

\section{Relationship Between Methylation and Expression of the Real Hub Gene}

We examined the relationship between the real hub gene's expression level and its methylation status to illuminate the potential cause of aberrant BLM expression in CCA. With the help of DiseaseMeth version 2.0, it revealed that the mean methylation level of BLM was significantly lower in CCA than in paracancerous normal tissue (Figure 6E). Additionally, we discovered that many methylation sites in the DNA sequence of gene BLM had a negative correlation with BLM expression using MEXPRESS (Figure 6F).

\section{GSEA of Real Hub Gene}

To determine the potential function of BLM in CCA, GSEA was performed to seek significant pathways enriched in the sample group with high BLM expression. Nine gene sets, "BASE EXCISION REPAIR," "CELL CYCLE," "DNA REPLICATION," "HOMOLOGOUS RECOMBINATION"” "MISMATCH REPAIR," "OOCYTE MEIOSIS," "PROGESTERONE MEDIATED OOCYTE MATURATION," "SPLICEOSOME," and "NUCLEOTIDE EXCISION REPAIR" were enriched (FDR < 0.05 ; Figure 6G). Overall, these gene sets were tightly associated with cell proliferation.

\section{BLM Expression Level Is Upregulated in CCA Cell Lines and CCA Tissue Samples}

Through the previous analysis, BLM was identified as the only real hub gene. We further investigated its expression level in different cell lines. The experiment results indicated that cancer cells (QBC939 and RBE) expressed higher levels of BLM than normal cells (HiBEC) at both the mRNA and protein levels (Figures 7A,B). We then conducted immunohistochemistry (IHC) assay to assess BLM expression utilizing a CCA TMA (including 27 CCA tissues and 9 paracancerous samples). As presented in Figure 7C, expression levels of BLM showed higher levels in CCA tissues compared with paracancerous samples (staining index: non-cancer $=3.78 \pm 0.49$; cancer $=6.78 \pm 0.29)$.

\section{BLM Knockdown Inhibits the Proliferation and Migration of CCA Cells in vitro and Induced G2-Phase Arrest}

To study the function of BLM in vitro, CCA cell lines (QBC939 and RBE) were transfected with control siRNA (scramble) or BLM siRNA (si-RNA1 and si-RNA2). Via PCR and western blot analysis of the harvested cells, we confirmed that BLM expression was significantly knocked down in BLM siRNA transfected cells than that in control groups at both the mRNA (Figure 8A) and protein (Figure 8B) levels. Using the CCK8 assay, we analyzed the viability of BLM-downregulation cells. Our results demonstrated that the downregulation of BLM expression significantly restrained the proliferation of both cell lines (Figures 8C,D). Next, we also assessed the effect of BLM on CCA cell migration in vitro. The downregulation of BLM significantly inhibited the migration of QBC-939 and RBE cells (Figures 8E-I). These results suggest a promotive role of BLM in CCA cell proliferation and migration. Moreover, we checked whether BLM silencing has cell cycle arrest effect in CCA cell lines. Cell cycle analysis performed by flow cytometry revealed that downregulation of BLM increased the cell percentage in G2 phase and decreased those in G1 phase and S phase (Figures 8J-L). It indicates that BLM can promote the CCA progression by modulating the cell cycle. 
A

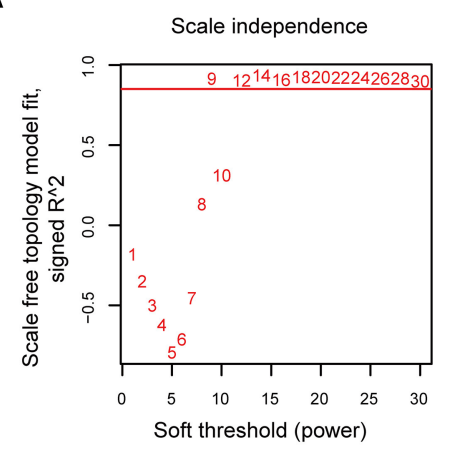

B

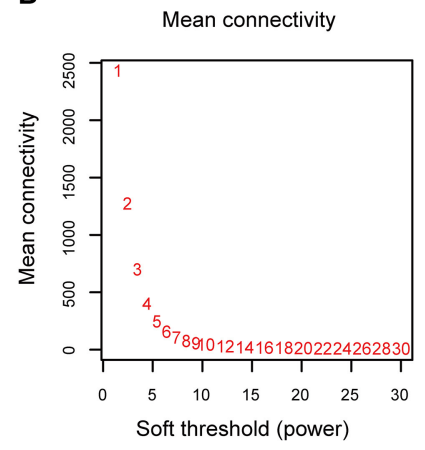

C

Cluster Dendrogram
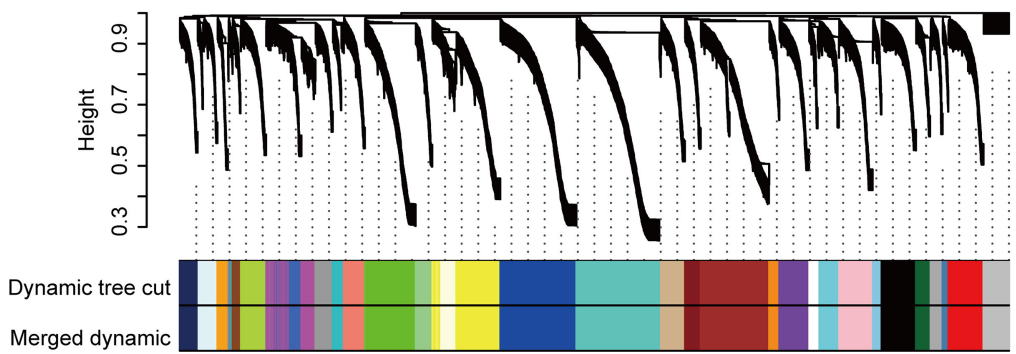

D

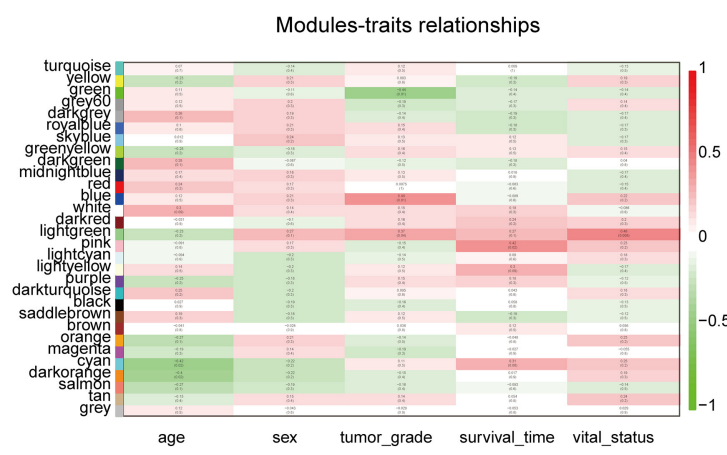

E

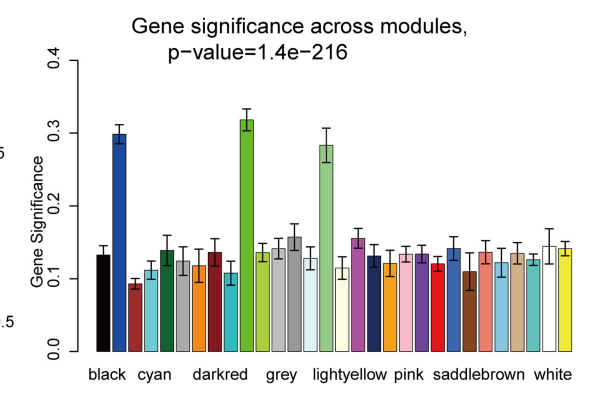

FIGURE 2 | WGCNA of DEGs in CCA samples. (A) Examination of the scale-free fit index for distinct soft-thresholding powers ( $\beta$ ). (B) Examination of the mean connectivity for distinct soft-thresholding powers. (C) Clustering dendrogram of filtered DEGs due to a dissimilarity measure. (D) Heatmap showing the connection between module eigengenes and five clinical traits of CCA. (E) Distribution of mean gene significance and errors in the modules correlated with tumor grade of CCA. WGCNA, weighted gene co-expression network analysis.

\section{DISCUSSION}

CCA is a highly malignant and heterogeneous neoplasm with a largely unknown molecular basis $(27,28)$. Because CCA is highly invasive and has no efficient screening methods, once diagnosed, only approximately one-third of patients are suitable for radical resection. However, even undergoing R0 resection, the disease recurrence rate is still high for patients with CCA, with an informed incidence of 60 and $80 \%$ at a median followup time of 2 and 5 years, respectively (29-31). For patients with late-stage CCA who have lost the chance of radical operation, the 5-year survival rate of non-surgical treatment is only $5 \%(28,32)$. Therefore, exploring the pathogenesis of
CCA and searching for key molecules closely associated with the occurrence, progression, and prognosis of the disease is particularly significant for the current diagnostic and treatment strategies of patients with CCA. In this study, first, we determined the blue module most relevant to the pathological grade of CCA by employing WGCNA. Five candidate genes were identified. After subsequent analyses, a real hub gene tightly related to the progression and prognosis of CCA was screened. Our findings may help to improve the ability of treatment decision-making, risk assessment, and prognosis prediction for patients with CCA.

The biological network can intuitively display the interrelationships between various functional elements in the biological system and provide an important platform for the 
A

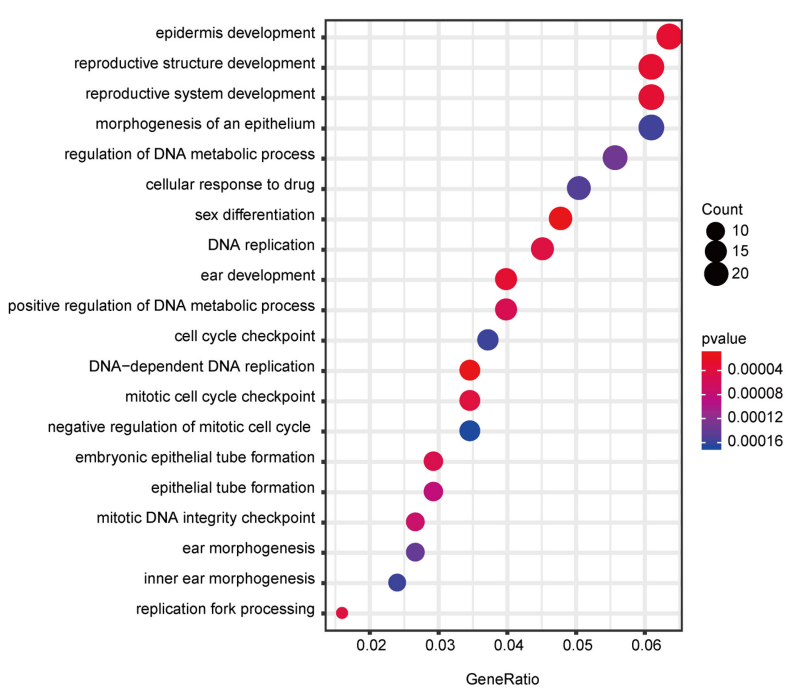

C

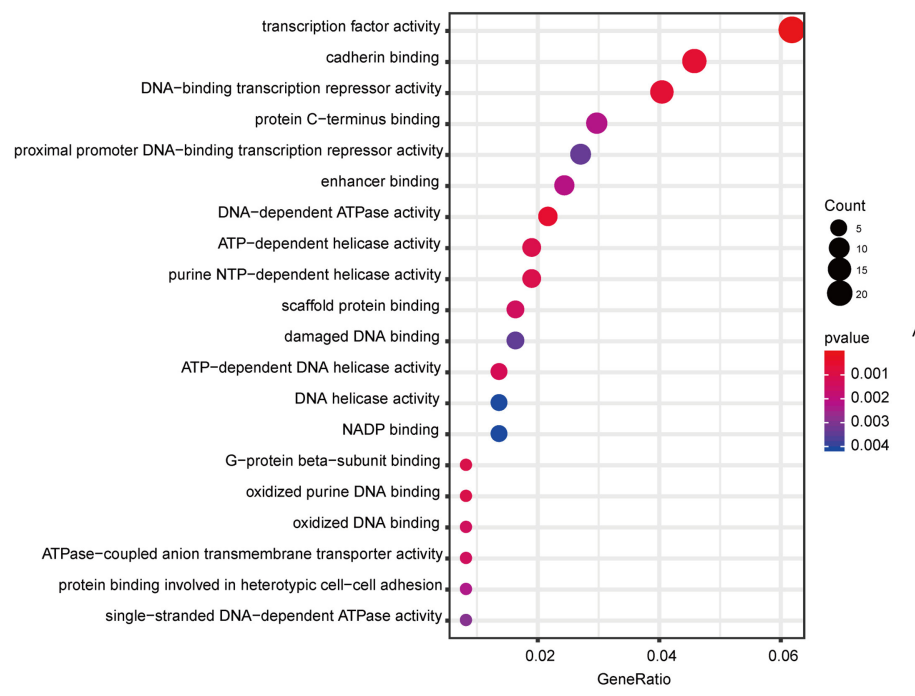

B

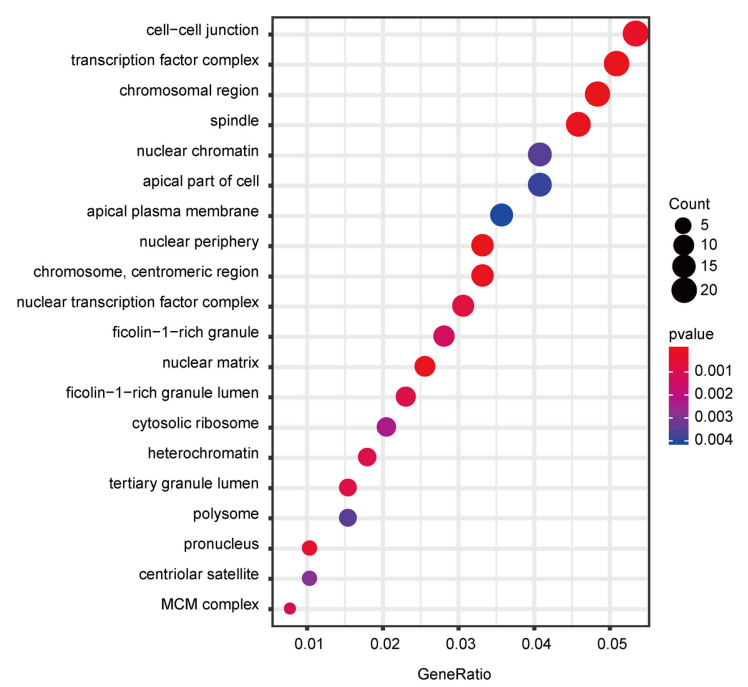

D

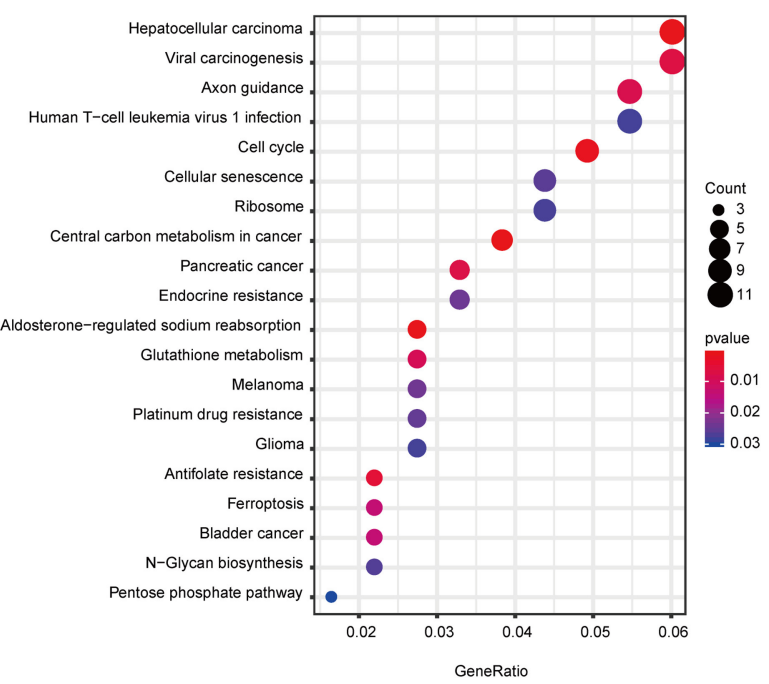

FIGURE 3 | Functional annotation of all the blue module genes. (A) GO terms for biological process. (B) GO terms for cellular component. (C) GO terms for molecular function. (D) KEGG enrichment pathway for genes in the blue module. GO, gene ontology; KEGG, Kyoto Encyclopedia of Genes and Genomes.

characteristics of the study objects at the system level $(33,34)$. In combination with the traditional one-dimensional molecular biology research methods, network analysis methods can more accurately illustrate the characteristics of biological systems (35). Gene co-expression network research, as an essential component of biological network research, can aid in obtaining multiple gene modules based on the gene expression profile by analyzing the expression correlation of multiple genes and in studying the association between genes and external traits at the gene module level $(36,37)$. We conducted a WGCNA to identify the co-expression modules correlated to the CCA progression. A total of 5,378 filtered DEGs were utilized to build a co-expression network, and 29 modules were screened. Loss of differentiation is a common event in tumor progression, and a high histological grade largely has an intimate correlation with an unfavorable prognosis (38). Here, it was found that the blue module had the highest correlation with tumor grade, which means that blue module genes are closely related to clinical traits. GO analysis can describe how genes act in biological systems and can put these descriptions in a computable format, while a KEGG pathway enrichment analysis displays higher-order functional significance and indicates the value of the cell or the organism from its genome data $(39,40)$. In line with the published data, the enrichment of blue module genes in several GO terms, such as DNA replication, cell-cell junction, mitotic cell cycle checkpoint, and cadherin binding, confirms their involvement in 
A

\section{Module membership vs. gene significance cor $=0.54, p=1.4 e-34$}

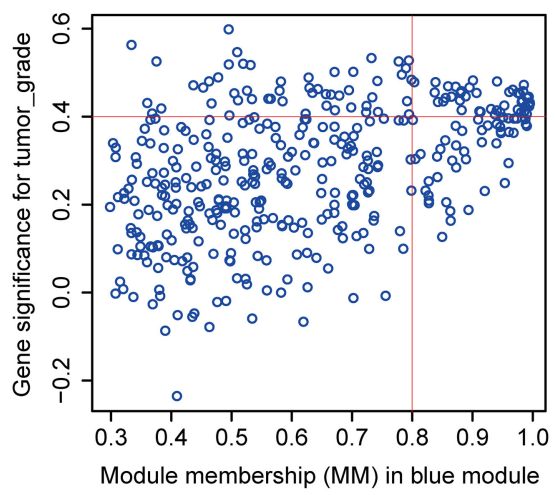

C

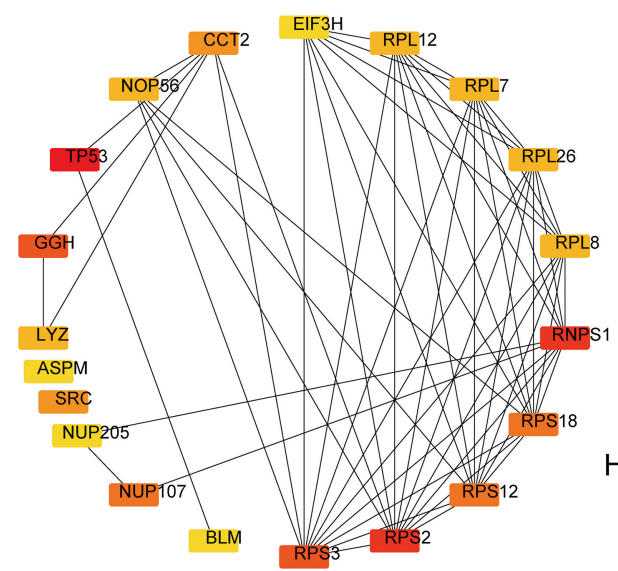

B

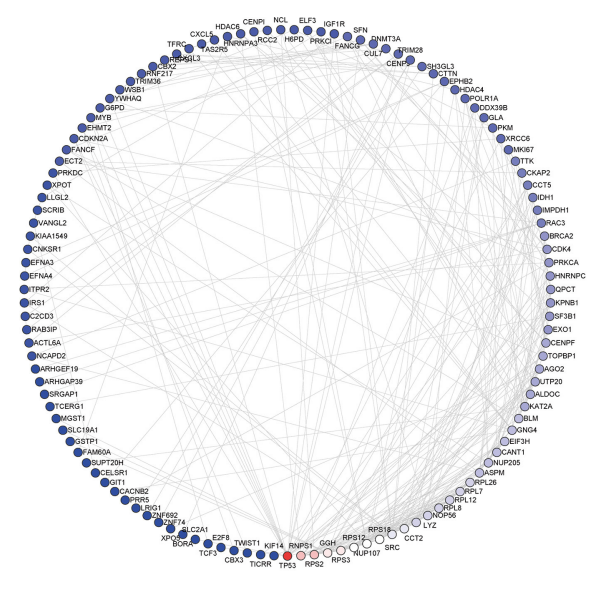

D

Hub genes in co-expression network

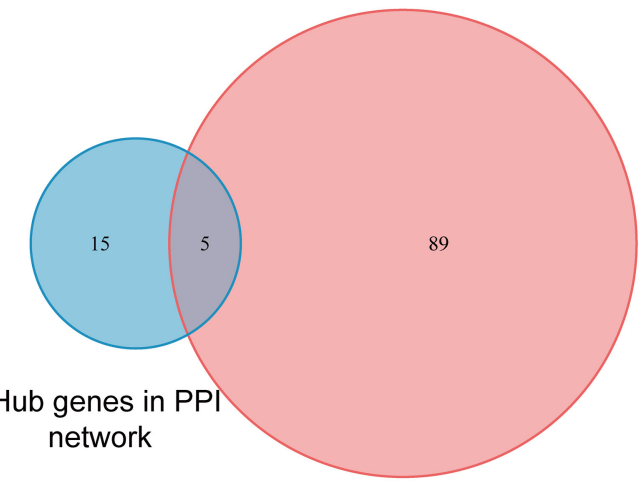

FIGURE 4 | PPI network and hub gene detection. (A) Scatter plot of module eigengenes in the blue module. (B) PPI network for blue module gene. The color intensity of each node was proportional to the connectivity degree in the PPI network (highest connectivity degree in red and lowest connectivity degree in blue). (C) Top 20 hub nodes screened by the cytoHubba plugin of Cytoscape software. (D) Identification of candidate genes in the PPI network and co-expression network. PPI, Protein-protein interaction.

the development of CCA $(41,42)$. Moreover, the KEGG pathway enrichment results of the identified module genes also indicate their involvement in CCA pathogenesis. For example, hepatitis $\mathrm{C}$ virus (HCV) infection is identified as an important risk factor for the development of CCA (43); recent progress has shown that cellular senescent markers can distinguish cholangiocellular carcinoma from duct adenoma, implying a possible role in the pathophysiology of CCA $(44,45)$. Additionally, cell cycle changes are particularly important for the growth of malignant tumors (42). According to the GO and KEGG enrichment results, we propose that these blue module genes are closely related to the cell cycle regulation and CCA development.

A study on the cellular function requires a deep understanding of functional interactions between the expressed proteins. The online STRING database can collect and integrate the cellular function data from the investigated and predicted PPI information for a great number of organisms (19). By constructing a PPI network based on the STRING database, we can study the interaction of proteins expressed by a group of genes and identify the key network proteins among them. In addition, hub genes of the co-expression network are characterized as highly related to the clinical traits of CCA samples in TCGA. A total of five candidates (BLM, RPS3, GGH, NUP107, and CCT2) were distinguished, which were common hub genes in both the co-expression network and PPI network of the blue module. All of them were reported to exert important roles in the pathogenesis of some carcinomas (46-50). Here, we carried out an expression pattern analysis and a survival analysis to explore the real hub genes, and BLM with high diagnostic and prognostic value was ultimately identified. Meanwhile, in the cell and tissue levels, we clarified the expression of the real hub gene, and the results further confirmed that BLM possesses completely different expression patterns in CCA compared with the non-tumor control.

The RecQ helicase family, which includes RECQ1, RECQ4, RECQ5, Werner protein (WRN), and BLM, plays an 
A
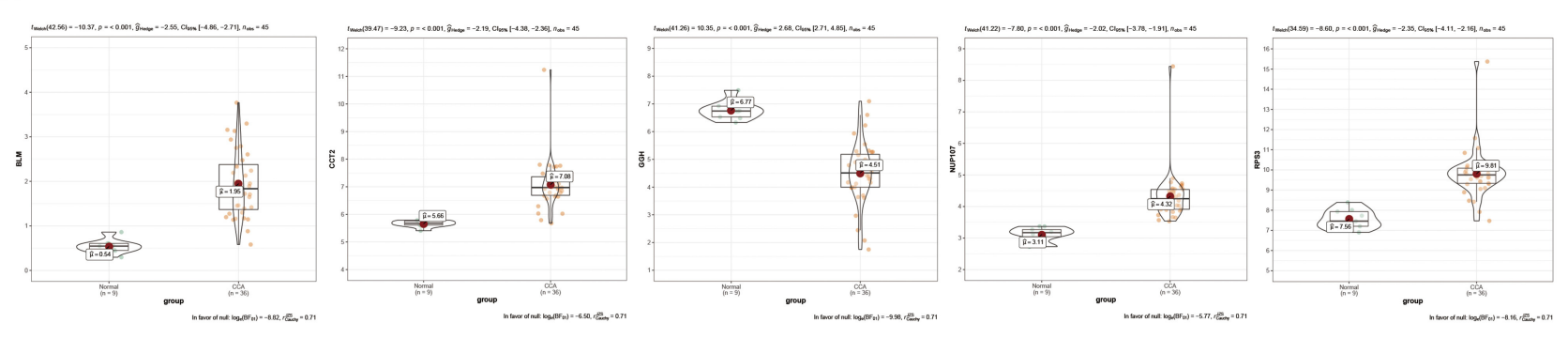

B
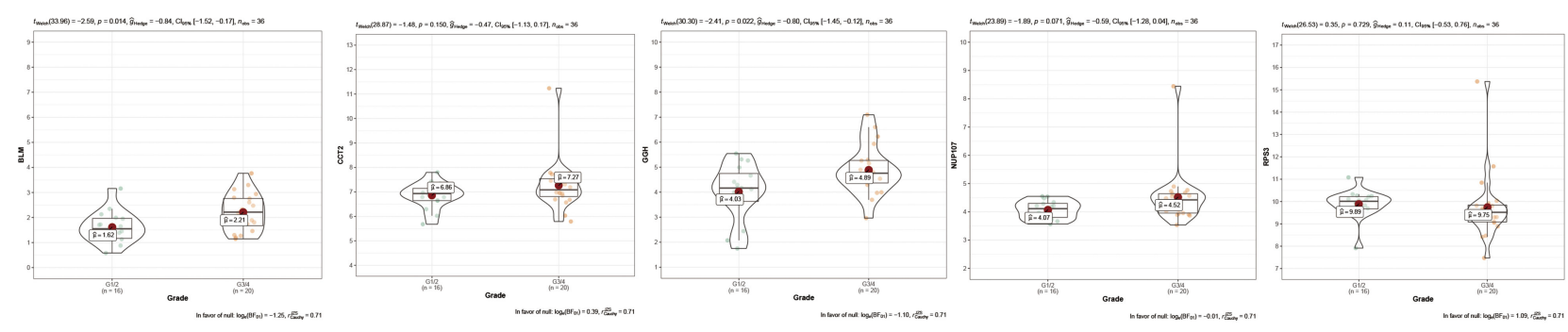

C
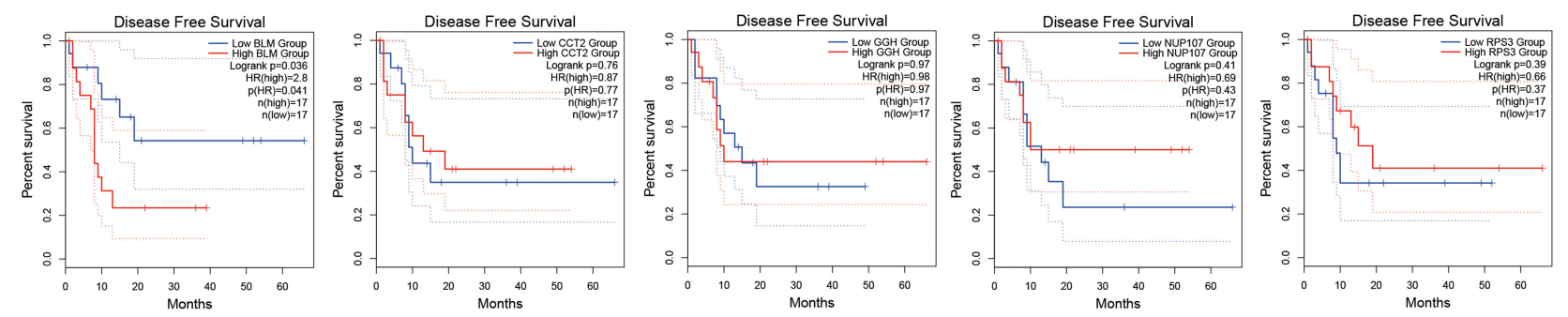

D
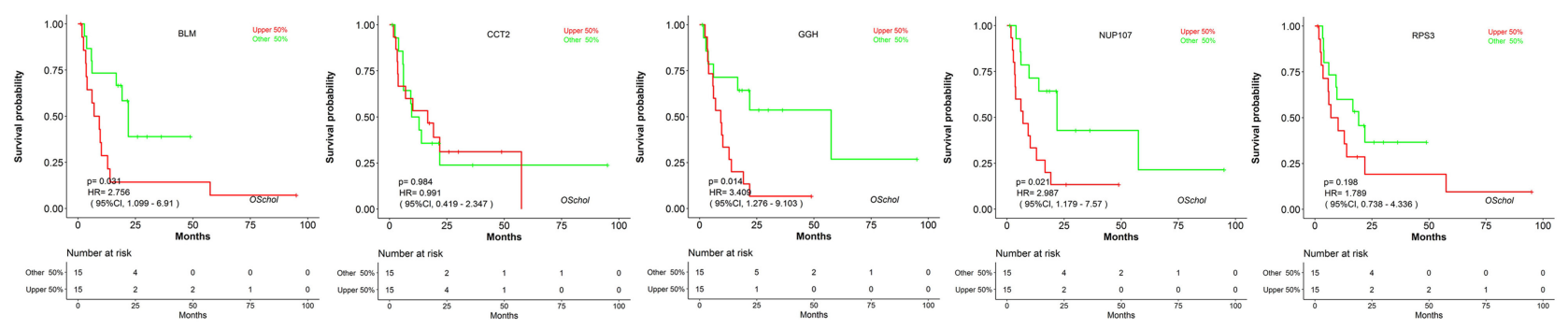

FIGURE 5 | Validation of candidate hub genes in the TCGA-CHOL data set. (A) bloom syndrome helicase (BLM), CCT2, GGH, NUP107, and RPS3 gene expression contrast between CCA and non-tumorous adjacent samples. (B) Expression of BLM, CCT2, GGH, NUP107, and RPS3 in CCA samples with different pathological grades. (C) Association between BLM, CCT2, GGH, NUP107, and RPS3 expression and disease-free survival time in the TCGA-CHOL data set. The red line indicates samples with high gene expression (above the median value), and the blue line designates the samples with lowly expressed genes (below the median value). (D) Association between BLM, CCT2, GGH, NUP107, and RPS3 expression and disease-free survival time in the data set GSE107943. The red line indicates samples with high gene expression (above the median value), and the green line designates the samples with lowly expressed genes (below the median value).

indispensable role in DNA replication, repair, RNA transcription, and telomere maintenance $(51,52)$. Germ cell mutations in these genes can lead to inherited diseases characterized by premature aging and/or cancer propensity, suggesting a pivotal role of the RecQ family in genome stability (53). For example, WRN, BLM, and RECQ4 are closely associated with the 
A

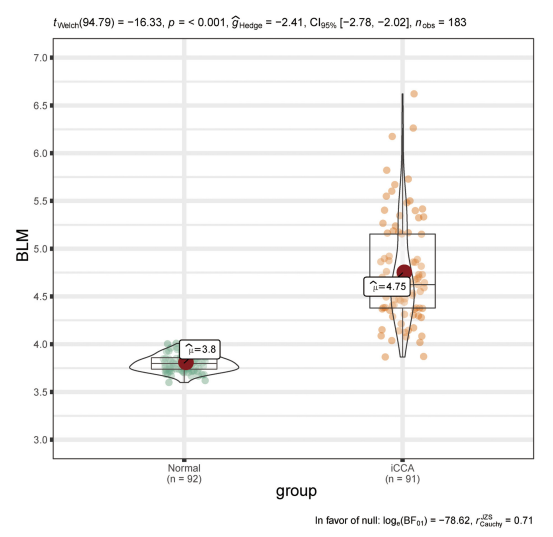

B

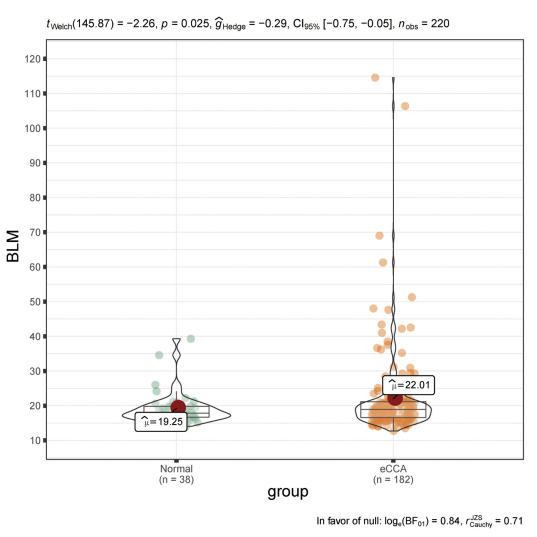

C

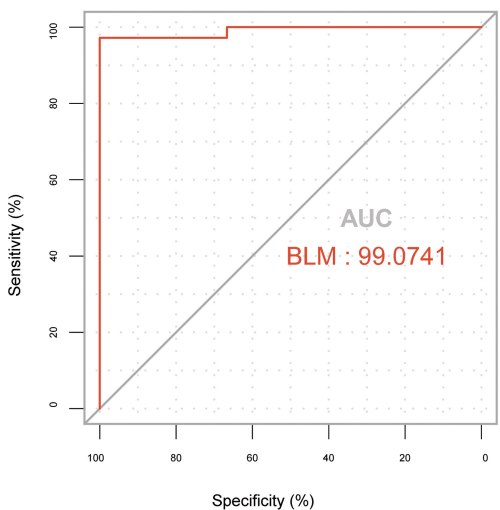

D

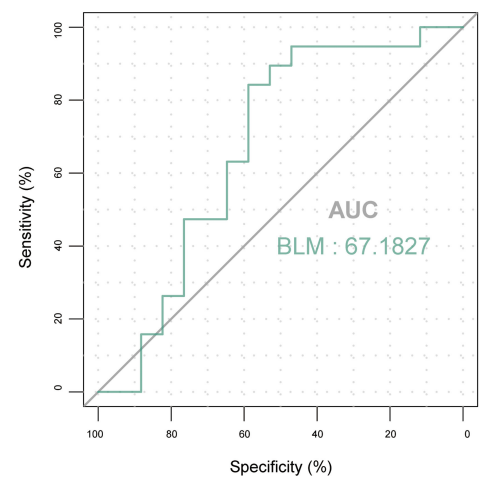

$\mathbf{F}$
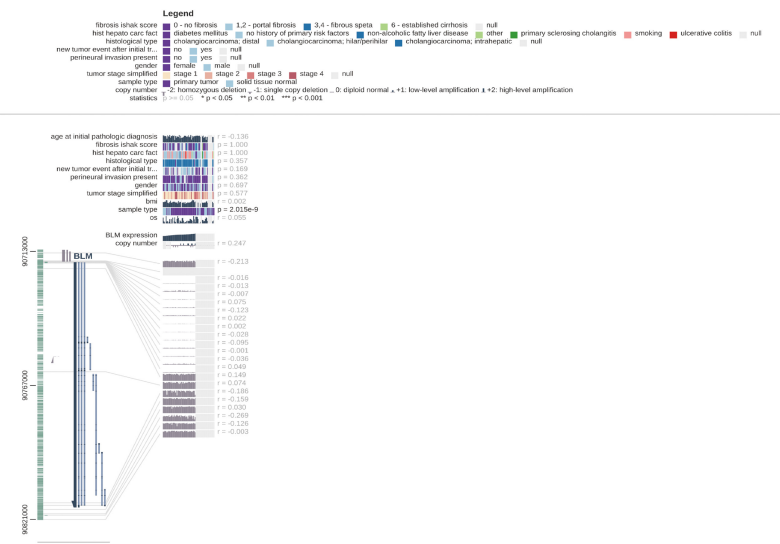

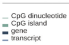

E

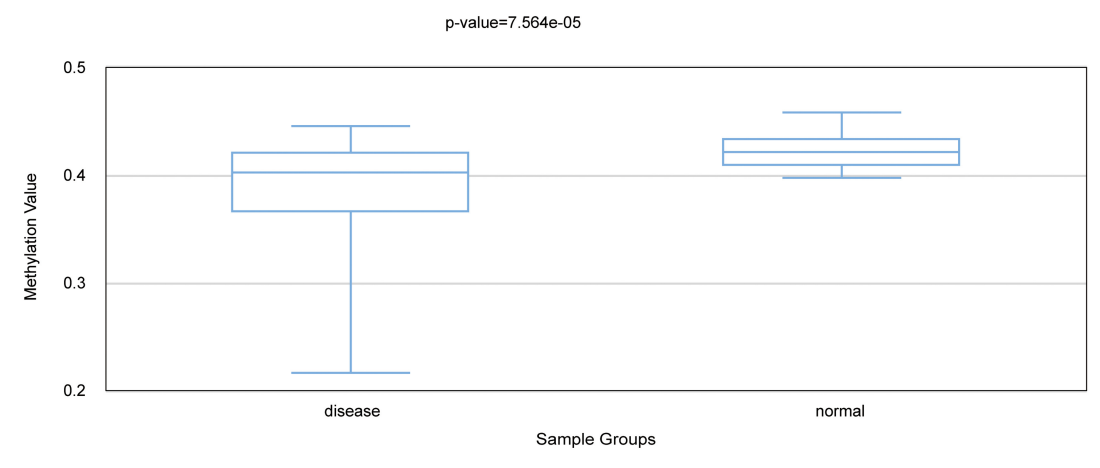

G

FIGURE 6 | The expression of BLM in the external data sets of GSE76297 and GSE132305, the methylation level of BLM, the value of BLM in the diagnosis and prediction of recurrence, and gene set enrichment analysis (GSEA). (A) BLM gene expression differences between iCCA and adjacent non-tumor tissues of the iCCA data set GSE76297. (B) BLM gene expression differences between eCCA and adjacent non-tumor tissues of the eCCA data set GSE132305. ROC curves and AUC statistics were used to assess the capacity of BLM for CCA diagnosis (C) and to distinguish recurrent and non-recurrent CCA (D). (E) The methylation levels of BLM in CCA and paracarcinoma tissue were examined by using DiseaseMeth 2.0. (F) The methylation sites of BLM and its associations with gene expression were visualized by using MEXPRESS. (G) GSEA using the TCGA-CHOL data set and statistically 9 significant functional gene sets enriched in CCA samples with high BLM expression were listed. iCCA, intrahepatic cholangiocarcinoma; eCCA, extrahepatic cholangiocarcinoma; ROC, receiver operating characteristic; AUC, area under the curve. 
A

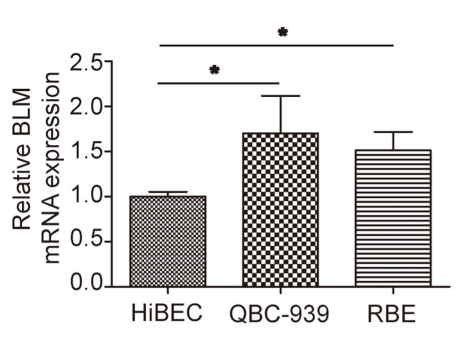

C

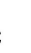

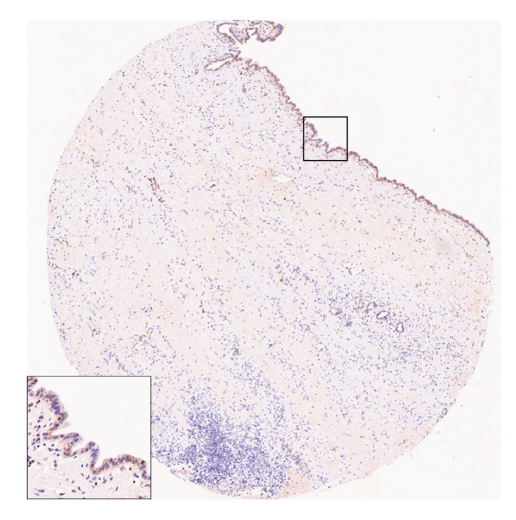

paracancerous normal tissues
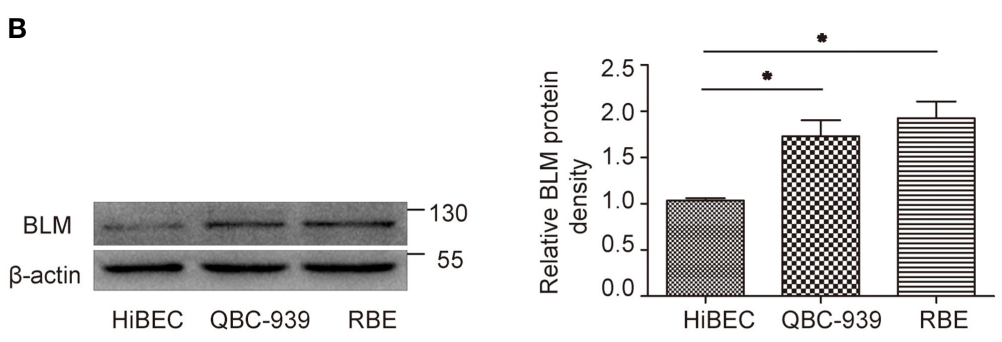

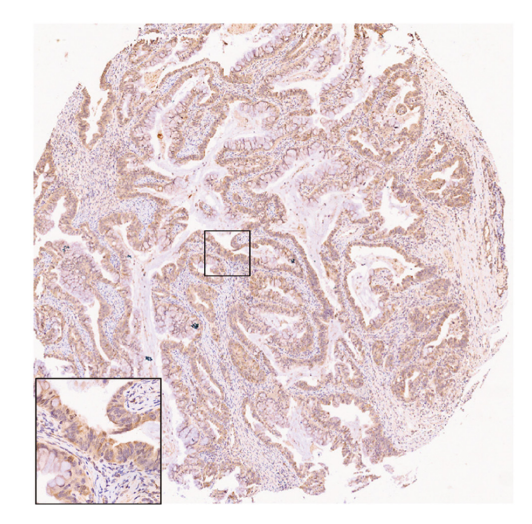

tumor tissues

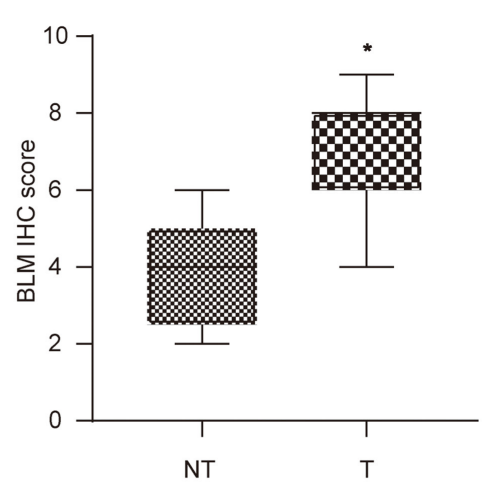

FIGURE 7 | BLM expression was increased in CCA. (A,B) qRT-PCR (A) and western blot (B) analysis of BLM expression in human biliary epithelial cell lines (HiBECs), QBC-939 cells, and RBE cells. (C) Representative BLM immunohistochemistry (IHC) staining and score for NT and T samples. T, tumoral tissue; NT, corresponding adjacent non-tumoral tissues. Data are represented as the mean \pm SEM of 3 independent experiments. ${ }^{*} p<0.05$ vs. the control group.

Werner syndrome, Bloom syndrome, and Rothmund Thomson syndrome, respectively, for which there are currently no effective therapies (54). Meanwhile, recent studies have demonstrated that RecQ helicases are also involved in the pathogenesis of multiple human sporadic tumors. RECQ1 may serve as a vital mediator in promoting the non-small cell lung cancer progression via the regulation in epithelial to mesenchymal transition (EMT) (55). Lieb et al. proved that the WRN inactivation selectively decreased the viability of microsatellite instability-high (MSI-H) colorectal and endometrial cancer cell lines (56). Moreover, RECQ4 overexpression accelerated the DNA replication rate and reduced chemosensitivity in breast cancer, thus promoting the tumor progression in established breast cancers (57). Patients with Bloom syndrome, who have BLM germ-line mutation resulting in striking decreases in both mRNA and protein expression levels of BLM, are apt to develop diverse malignancies including breast cancer, prostate cancer, and lung cancer $(52,58)$. Furthermore, previous studies have supposed that BLM serves as a cancer suppressor through targeting the proto-oncogene c-MYC, and recent researches also proposed that a series of malignancies are associated with the overexpression of the MYC gene and loss of BLM function $(54,59)$. Chandra et al. further confirmed this view in their research, which suggested that BLM indeed promoted the degradation of Myc proto-oncogene protein and subsequently led to an obstruction in the MYC-dependent induction of tumors (60). Interestingly, recent studies have also demonstrated that BLM is highly expressed in multiple cancer tissues and even acts as a novel cancer biomarker. For example, Chen et al. showed that BLM protein could promote cell proliferation and inhibit apoptosis via activating AKT signaling and downregulating the PTEN expression in prostate cancer cells (61); Arora et al. via analyzing molecular profiling in a large cohort of 1,980 breast cancer samples, found that BLM mRNA overexpression was linked to a poor breast cancer-specific survival and that high cytoplasmic BLM indicated aggressive phenotypes (62). Our current research has revealed that BLM expression is increased in CCA tissues compared with paracancerous samples, which has been further verified in assays in vitro, and high BLM expression is correlated with the tumor progression and adverse prognosis. Moreover, we found that BLM silencing represses the CCA cell proliferation and migration in vitro. More recently, the dysregulation of DNA methylation patterns has been increasingly perceived as a pivotal cellular procedure during both the initiation and progression of oncogenesis $(63,64)$. DNA hypomethylation of genome-wide regions, especially $\mathrm{CpG}$ islands, is a common epigenetic remodeling in CCA and is in 
A

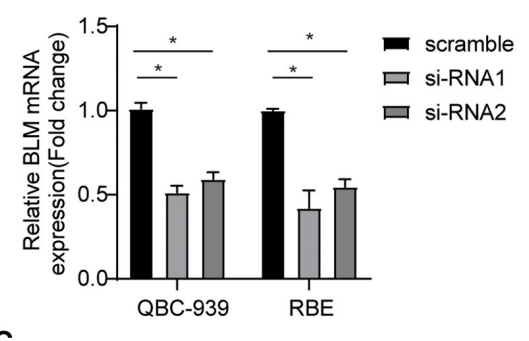

C

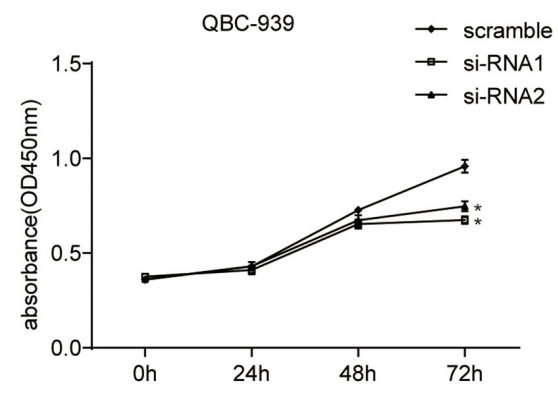

F

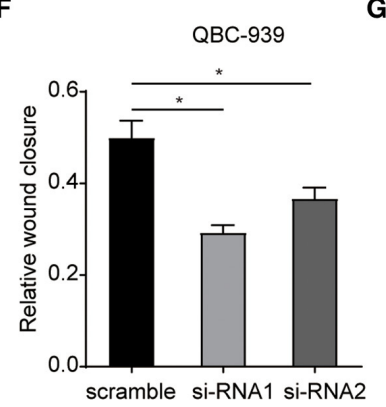

G

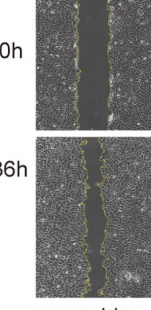

scramble
B

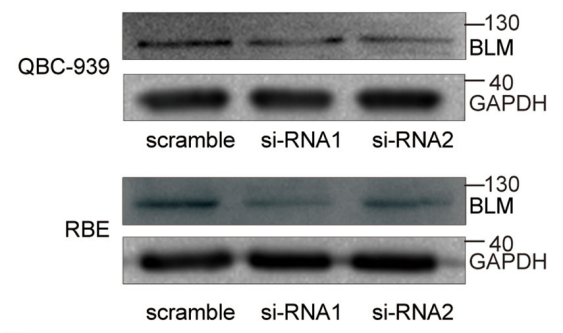

D

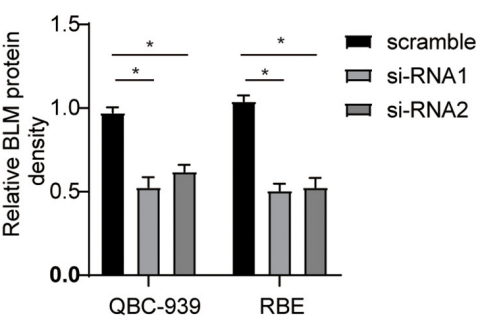

E

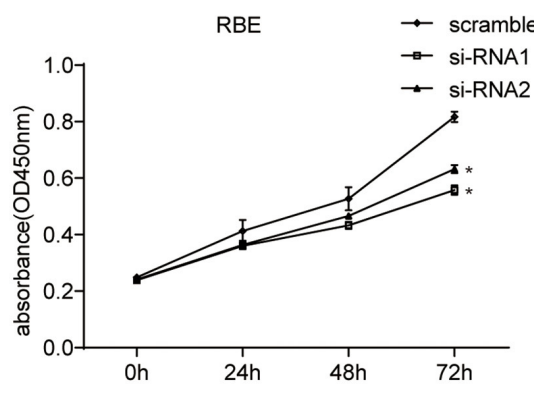

H
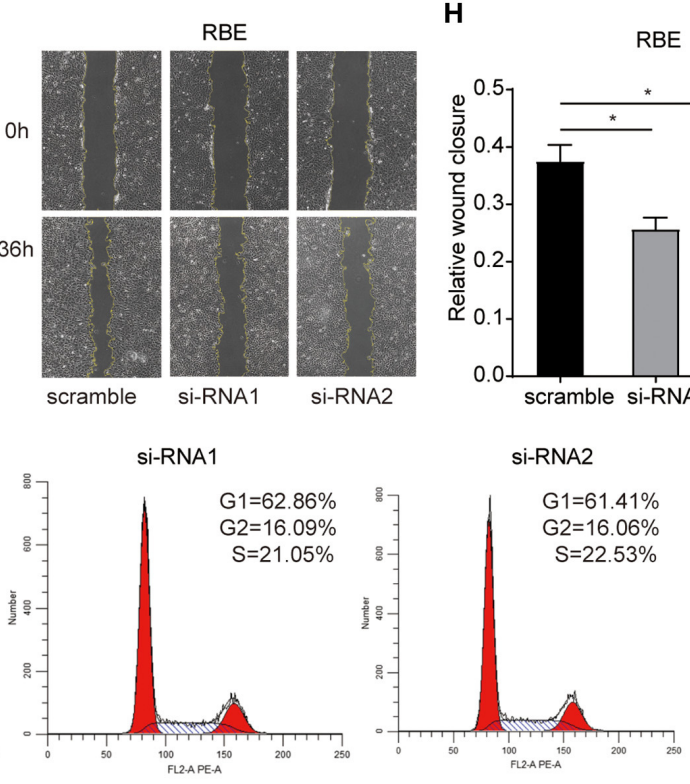

K

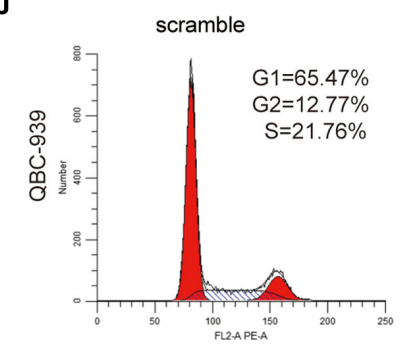

si-RNA1
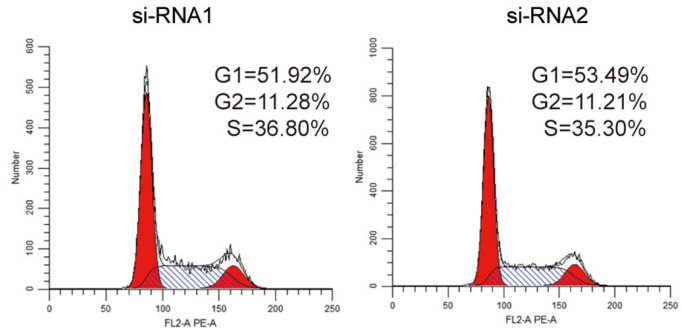

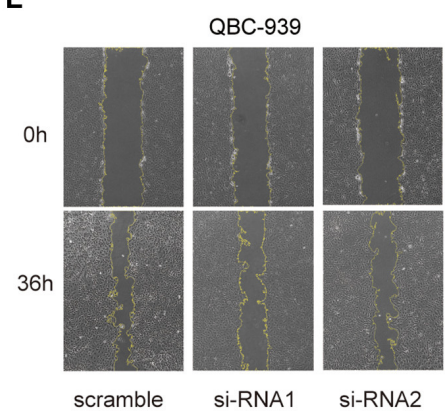

I
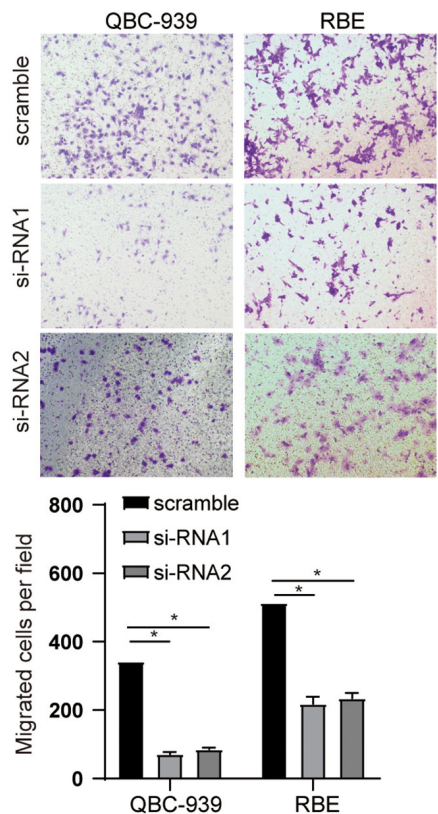

L

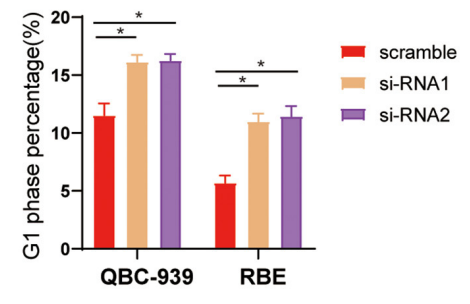

FIGURE 8 | BLM silencing inhibited the proliferation and migration of cholangioca-rcinoma cells and induced cell cycle arrest. (A) qRT-PCR validation of BLM knockdown. (B) WB validation of BLM knockdown. (C,D) CCK8 assay analysis of the proliferation of QBC-939 cells (C) and RBE cells (D) transfected with BLM small interfering RNA (siRNA) (si-RNA1 and si-RNA2) or control siRNA (scramble). (E-H) The effects of BLM knockdown on QBC939 cell and RBE cell migration ability were assessed by wound-healing assay; representative photographs of wound-healing assay (E,G), and quantitative results shown as relative migration rates (F,H). (I) The effects of BLM knockdown on QBC939 cell and RBE cell migration were evaluated by Tanswell assay. Representative photographs of the transwell assay (upper 
FIGURE 8 | panel) and quantitative results shown as number of migrated cells per field (lower panel). (J) Flow cytometric analysis of the cell cycle with BLM knockdown and control group in RBE cells. (K) Flow cytometric analysis of the cell cycle with BLM knockdown and control group in QBC-939 cells. (L) G2-phase analysis of the cell cycle with BLM knockdown and control cells. Data are represented as the mean \pm SEM of 3 independent experiments. ${ }^{*} p<0.05$ vs. control group.

correlation to the activation of some proto-oncogenes and the existence of chromosomal instability $(65,66)$. By using the online tools DiseaseMeth 2.0 and MEXPRESS, we also investigated the DNA methylation status that possibly resulted in the abnormal expression of BLM in CCA. BLM methylation was reduced in cancer tissues compared with the adjacent normal samples, which was in accordance with the observed upregulation of this real hub gene in CCA. Then, GSEA was executed to explore the possible pathogenesis of BLM in CCA. The results showed that various cell cycle-related KEGG pathways, such as the CELL CYCLE pathway, MISMATCH REPAIR pathway, and DNA REPLICATION pathway, were enriched in the high-expression group of BLM, indicating their contribution to CCA cell proliferation, and in vitro experiments results further confirmed the vital role of BLM in regulating the cell cycle of CCA.

\section{CONCLUSION}

In summary, our present study attempted to explore potential molecular mechanisms in CCA by employing a range of bioinformatics analyses. We identified a real hub gene (BLM) that might cause the progression and poor prognosis of CCA, and BLM might affect these biological processes by regulating the cell cycle process. All these findings contribute to the clinical diagnosis, treatment, and prognostic evaluation of CCA. Next, we will perform experimental research to explore the specific molecular biological mechanisms underlying the involvement of BLM in modulating the CCA occurrence and development.

\section{DATA AVAILABILITY STATEMENT}

The datasets presented in this study can be found in online repositories. The names of the repository/repositories and

\section{REFERENCES}

1. Abdel-Rahman O, Elsayed Z, Elhalawani H. Gemcitabine-based chemotherapy for advanced biliary tract carcinomas. Cochrane Database Syst Rev. (2018) 4:CD011746. doi: 10.1002/14651858.CD011746.pub2

2. Esnaola NF, Meyer JE, Karachristos A, Maranki JL, Camp ER, Denlinger CS. Evaluation and management of intrahepatic and extrahepatic cholangiocarcinoma. Cancer. (2016) 122:1349-69. doi: 10.1002/cncr.29692

3. Blechacz B, Komuta M, Roskams T, Gores GJ. Clinical diagnosis and staging of cholangiocarcinoma. Nat Rev Gastroenterol Hepatol. (2011) 8:51222. doi: $10.1038 /$ nrgastro.2011.131

4. Patel T. Cholangiocarcinoma-controversies and challenges. Nat Rev Gastroenterol Hepatol. (2011) 8:189-200. doi: 10.1038/nrgastro.2011.20

5. Gatta G, Capocaccia R, Botta L, Mallone S, De Angelis R, Ardanaz E, et al. Burden and centralised treatment in Europe of rare tumours: results of RARECAREnet-a population-based study. Lancet Oncol. (2017) 18:102239. doi: 10.1016/S1470-2045(17)30445-X accession number(s) can be found at: https://www.ncbi.nlm. nih.gov/geo/, GSE76297, GSE132305, https://portal.gdc.cancer. gov/, TCGA-CHOL.

\section{ETHICS STATEMENT}

The studies involving human participants were reviewed and approved by Shanghai Chaoshi Biotech Company Ethics Committee Shanghai Outdo Biotech Company, Shanghai, China. The patients/participants provided their written informed consent to participate in this study.

\section{AUTHOR CONTRIBUTIONS}

ZS conceived the study plan and contributed to the revision of the final manuscript. XD and $\mathrm{CZ}$ performed the experiments, analyzed the data, and finished the manuscript writing. CY, WW, $\mathrm{XY}, \mathrm{DX}$, and XZ participated in data collection and literature search. QZ contributed to the manuscript writing and data interpretation. ML guided the cell experiments. All authors contributed to the article and approved the submitted version.

\section{FUNDING}

This study was supported by the research grants from the National Natural Science Foundation of China to ZS (Grant Nos. 81974040 and 81670374) and ML (Grant No. 81874208).

\section{ACKNOWLEDGMENTS}

We thank all our colleagues working in the laboratory of General Surgery, Union Hospital, Tongji Medical College, Huazhong University of Science, and Technology, for their help and support.

6. Lazaridis KN, Gores GJ. Cholangiocarcinoma. Gastroenterology. (2005) 128:1655-67. doi: 10.1053/j.gastro.2005.03.040

7. Yang XQ, Xu YF, Guo S, Liu Y, Ning SL, Lu XF, et al. Clinical significance of nerve growth factor and tropomyosin-receptor-kinase signaling pathway in intrahepatic cholangiocarcinoma. World J Gastroenterol. (2014) 20:407684. doi: 10.3748/wjg.v20.i14.4076

8. Squadroni M, Tondulli L, Gatta G, Mosconi S, Beretta G, Labianca R. Cholangiocarcinoma. Crit Rev Oncol Hematol. (2017) 116:11-31. doi: 10.1016/j.critrevonc.2016.11.012

9. Werner T. Bioinformatics applications for pathway analysis of microarray data. Curr Opin Biotechnol. (2008) 19:50-4. doi: 10.1016/j.copbio.2007.11.005

10. Mutz KO, Heilkenbrinker A, Lonne M, Walter JG, Stahl F. Transcriptome analysis using next-generation sequencing. Curr Opin Biotechnol. (2013) 24:22-30. doi: 10.1016/j.copbio.2012.09.004

11. Pavlopoulos GA, Wegener AL, Schneider R. A survey of visualization tools for biological network analysis. BioData Min. (2008) 1:12. doi: 10.1186/1756-0381-1-12 
12. van Dam S, Vosa U, van der Graaf A, Franke L, de Magalhaes JP. Gene coexpression analysis for functional classification and gene-disease predictions. Brief Bioinform. (2018) 19:575-92. doi: 10.1093/bib/bbw139

13. Miller JA, Horvath S, Geschwind DH. Divergence of human and mouse brain transcriptome highlights Alzheimer disease pathways. Proc Natl Acad Sci USA. (2010) 107:12698-703. doi: 10.1073/pnas.0914257107

14. Carlson MR, Zhang B, Fang Z, Mischel PS, Horvath S, Nelson SF. Gene connectivity, function, and sequence conservation: predictions from modular yeast co-expression networks. BMC Genomics. (2006) 7:40. doi: 10.1186/1471-2164-7-40

15. Colaprico A, Silva TC, Olsen C, Garofano L, Cava C, Garolini D, et al. TCGAbiolinks: an R/Bioconductor package for integrative analysis of TCGA data. Nucleic Acids Res. (2016) 44:e71. doi: 10.1093/nar/gkv1507

16. Langfelder P, Horvath S. WGCNA: an R package for weighted correlation network analysis. BMC Bioinformatics. (2008) 9:559. doi: 10.1186/1471-2105-9-559

17. Yu G, Wang LG, Han Y, He QY. clusterProfiler: an R package for comparing biological themes among gene clusters. OMICS. (2012) 16:2847. doi: 10.1089/omi.2011.0118

18. Walter W, Sanchez-Cabo F, Ricote M. GOplot: an R package for visually combining expression data with functional analysis. Bioinformatics. (2015) 31:2912-4. doi: 10.1093/bioinformatics/btv300

19. Szklarczyk D, Morris JH, Cook H, Kuhn M, Wyder S, Simonovic M, et al. The STRING database in 2017: quality-controlled protein-protein association networks, made broadly accessible. Nucleic Acids Res. (2017) 45:D3628. doi: 10.1093/nar/gkw937

20. Shannon P, Markiel A, Ozier O, Baliga NS, Wang JT, Ramage $\mathrm{D}$, et al. Cytoscape: a software environment for integrated models of biomolecular interaction networks. Genome Res. (2003) 13:2498-504. doi: 10.1101/gr.1239303

21. Xiong Y, Wei Y, Gu Y, Zhang S, Lyu J, Zhang B, et al. DiseaseMeth version 2.0: a major expansion and update of the human disease methylation database. Nucleic Acids Res. (2017) 45:D888-95. doi: 10.1093/nar/gkw1123

22. Koch A, Jeschke J, Van Criekinge W, van Engeland $M$, De Meyer T. MEXPRESS update 2019. Nucleic Acids Res. (2019) 47:W561-5. doi: 10.1093/nar/gkz445

23. Robin X, Turck N, Hainard A, Tiberti N, Lisacek F, Sanchez JC, et al. pROC: an open-source package for $\mathrm{R}$ and $\mathrm{S}+$ to analyze and compare ROC curves. BMC Bioinformatics. (2011) 12:77. doi: 10.1186/1471-2105-12-77

24. Subramanian A, Tamayo P, Mootha VK, Mukherjee S, Ebert BL, Gillette MA, et al. Gene set enrichment analysis: a knowledge-based approach for interpreting genome-wide expression profiles. Proc Natl Acad Sci USA. (2005) 102:15545-50. doi: 10.1073/pnas.0506580102

25. Livak KJ, Schmittgen TD. Analysis of relative gene expression data using realtime quantitative PCR and the 2(-Delta Delta C(T)) Method. Methods. (2001) 25:402-8. doi: 10.1006/meth.2001.1262

26. Wang Q, An Y, Wang F, Zhang G, Zhang L, Dong H, et al. OSchol: an online consensus survival web server for cholangiocarcinoma prognosis analysis. HPB. (2020). doi: 10.1016/j.hpb.2020.08.011. [Epub ahead of print].

27. Razumilava N, Gores GJ. Cholangiocarcinoma. Lancet. (2014) 383:216879. doi: 10.1016/S0140-6736(13)61903-0

28. Aljiffry M, Abdulelah A, Walsh M, Peltekian K, Alwayn I, Molinari M. Evidence-based approach to cholangiocarcinoma: a systematic review of the current literature. J Am Coll Surg. (2009) 208:134-47. doi: 10.1016/j.jamcollsurg.2008.09.007

29. Weber SM, Ribero D, O’Reilly EM, Kokudo N, Miyazaki M, Pawlik TM. Intrahepatic cholangiocarcinoma: expert consensus statement. HPB. (2015) 17:669-80. doi: 10.1111/hpb.12441

30. Rahnemai-Azar AA, Pandey P, Kamel I, Pawlik TM. Monitoring outcomes in intrahepatic cholangiocarcinoma patients following hepatic resection. Hepat Oncol. (2016) 3:223-39. doi: 10.2217/hep-2016-0009

31. Hyder O, Hatzaras I, Sotiropoulos GC, Paul A, Alexandrescu $\mathrm{S}$, Marques $\mathrm{H}$, et al. Recurrence after operative management of intrahepatic cholangiocarcinoma. Surgery. (2013) 153:8118. doi: 10.1016/j.surg.2012.12.005

32. Valle J, Wasan H, Palmer DH, Cunningham D, Anthoney A, Maraveyas A, et al. Cisplatin plus gemcitabine versus gemcitabine for biliary tract cancer. $N$ Engl J Med. (2010) 362:1273-81. doi: 10.1056/NEJMoa0908721
33. Furlong LI. Human diseases through the lens of network biology. Trends Genet. (2013) 29:150-9. doi: 10.1016/j.tig.2012.11.004

34. Barabasi AL, Oltvai ZN. Network biology: understanding the cell's functional organization. Nat Rev Genet. (2004) 5:101-13. doi: 10.1038/nrg1272

35. Barabasi AL, Gulbahce N, Loscalzo J. Network medicine: a network-based approach to human disease. Nat Rev Genet. (2011) 12:56-68. doi: 10.1038/nrg2918

36. Hirose O, Yoshida R, Imoto S, Yamaguchi R, Higuchi T, Charnock-Jones DS, et al. Statistical inference of transcriptional module-based gene networks from time course gene expression profiles by using state space models. Bioinformatics. (2008) 24:932-42. doi: 10.1093/bioinformatics/btm639

37. D'Haeseleer P, Liang S, Somogyi R. Genetic network inference: from coexpression clustering to reverse engineering. Bioinformatics. (2000) 16:70726. doi: 10.1093/bioinformatics/16.8.707

38. Dom G, Galdo VC, Tarabichi M, Tomas G, Hebrant A, Andry G, et al. 5-aza2 '-deoxycytidine has minor effects on differentiation in human thyroid cancer cell lines, but modulates genes that are involved in adaptation in vitro. Thyroid. (2013) 23:317-28. doi: 10.1089/thy.2012.0388

39. Kanehisa M, Goto S, Kawashima S, Okuno Y, Hattori M. The KEGG resource for deciphering the genome. Nucleic Acids Res. (2004) 32:D27780. doi: 10.1093/nar/gkh063

40. Ashburner M, Ball CA, Blake JA, Botstein D, Butler H, Cherry JM, et al. Gene ontology: tool for the unification of biology. The gene ontology consortium. Nat Genet. (2000) 25:25-9. doi: 10.1038/75556

41. Kourtidis A, Lu R, Pence LJ, Anastasiadis PZ. A central role for cadherin signaling in cancer. Exp Cell Res. (2017) 358:7885. doi: 10.1016/j.yexcr.2017.04.006

42. Kastan MB, Bartek J. Cell-cycle checkpoints and cancer. Nature. (2004) 432:316-23. doi: 10.1038/nature03097

43. Navas MC, Glaser S, Dhruv H, Celinski S, Alpini G, Meng F. Hepatitis $C$ virus infection and cholangiocarcinoma: an insight into epidemiologic evidences and hypothetical mechanisms of oncogenesis. Am J Pathol. (2019) 189:1122-32. doi: 10.1016/j.ajpath.2019.01.018

44. Sasaki M, Nakanuma Y. New concept: cellular senescence in pathophysiology of cholangiocarcinoma. Expert Rev Gastroenterol Hepatol. (2016) 10:62538. doi: 10.1586/17474124.2016.1133291

45. Sasaki M, Matsubara T, Kakuda Y, Sato Y, Nakanuma Y. Immunostaining for polycomb group protein EZH2 and senescent marker p16INK4a may be useful to differentiate cholangiolocellular carcinoma from ductular reaction and bile duct adenoma. Am J Surg Pathol. (2014) 38:3649. doi: 10.1097/PAS.0000000000000125

46. Zhao L, Cao J, Hu K, Wang P, Li G, He X, et al. RNA-binding protein RPS3 contributes to hepatocarcinogenesis by post-transcriptionally up-regulating SIRT1. Nucleic Acids Res. (2019) 47:2011-28. doi: 10.1093/nar/gky1209

47. Park SH, Jeong S, Kim BR, Jeong YA, Kim JL, Na YJ, et al. Activating CCT2 triggers Gli-1 activation during hypoxic condition in colorectal cancer. Oncogene. (2020) 39:136-50. doi: 10.1038/s41388-019-0972-6

48. Panier S, Maric M, Hewitt G, Mason-Osann E, Gali H, Dai A, et al. SLX4IP antagonizes promiscuous BLM activity during ALT maintenance. Mol Cell. (2019) 76:27-43.e11. doi: 10.1016/j.molcel.2019.07.010

49. Kuo WT, Tu DG, Chiu LY, Sheu GT, Wu MF. High pemetrexed sensitivity of docetaxel-resistant A549 cells is mediated by TP53 status and downregulated thymidylate synthase. Oncol Rep. (2017) 38:278795. doi: 10.3892/or.2017.5951

50. Hanafusa T, Mohamed AE, Kitaoka K, Ohue Y, Nakayama E, Ono T. Isolation and characterization of human lung cancer antigens by serological screening with autologous antibodies. Cancer Lett. (2011) 301:5762. doi: 10.1016/j.canlet.2010.10.024

51. Chang EY, Novoa CA, Aristizabal MJ, Coulombe Y, Segovia R, Chaturvedi R, et al. RECQ-like helicases Sgs1 and BLM regulate R-loop-associated genome instability. J Cell Biol. (2017) 216:3991-4005. doi: 10.1083/jcb.201703168

52. Croteau DL, Popuri V, Opresko PL, Bohr VA. Human RecQ helicases in DNA repair, recombination, and replication. Annu Rev Biochem. (2014) 83:519-52. doi: 10.1146/annurev-biochem-060713-03 5428

53. Sharma S, Doherty KM, Brosh RM Jr. DNA helicases as targets for anti-cancer drugs. Curr Med Chem Anticancer Agents. (2005) 5:18399. doi: $10.2174 / 1568011053765985$ 
54. Chu WK, Hickson ID. RecQ helicases: multifunctional genome caretakers. Nat Rev Cancer. (2009) 9:644-54. doi: 10.1038/nrc2682

55. Li L, Gao M, Song B, Zhang H, Wang Y. Effects of RECQ1 helicase silencing on non-small cell lung cancer cells. Biomed Pharmacother. (2016) 83:122732. doi: 10.1016/j.biopha.2016.07.053

56. Lieb S, Blaha-Ostermann S, Kamper E, Rippka J, Schwarz C, Ehrenhöfer-Wölfer K, et al. Werner syndrome helicase is a selective vulnerability of microsatellite instability-high tumor cells. eLife. (2019) 8:e43333. doi: 10.7554/eLife.43333

57. Arora A, Agarwal D, Abdel-Fatah TM, Lu H, Croteau DL, Moseley P, et al. RECQL4 helicase has oncogenic potential in sporadic breast cancers. J Pathol. (2016) 238:495-501. doi: 10.1002/path.4681

58. Cheok CF, Bachrati CZ, Chan KL, Ralf C, Wu L, Hickson ID. Roles of the Bloom's syndrome helicase in the maintenance of genome stability. Biochem Soc Trans. (2005) 33:1456-9. doi: 10.1042/BST0331456

59. Nesbit CE, Tersak JM, Prochownik EV. MYC oncogenes and human neoplastic disease. Oncogene. (1999) 18:3004-16. doi: 10.1038/sj.onc.1202746

60. Chandra S, Priyadarshini R, Madhavan V, Tikoo S, Hussain M, Mudgal R, et al. Enhancement of c-Myc degradation by BLM helicase leads to delayed tumor initiation. J Cell Sci. (2013) 126:3782-95. doi: 10.1242/jcs.124719

61. Chen $\mathrm{K}, \mathrm{Xu} \mathrm{H}$, Zhao J. Bloom syndrome protein activates AKT and PRAS40 in prostate cancer cells. Oxid Med Cell Longev. (2019) 2019:3685817. doi: 10.1155/2019/3685817

62. Arora A, Abdel-Fatah TM, Agarwal D, Doherty R, Moseley PM, Aleskandarany MA, et al. Transcriptomic and protein expression analysis reveals clinicopathological significance of bloom syndrome helicase (BLM) in breast cancer. Mol Cancer Ther. (2015) 14:1057-65. doi: 10.1158/1535-7163.MCT-14-0939
63. Jasek K, Kubatka P, Samec M, Liskova A, Smejkal K, Vybohova D, et al. DNA methylation status in cancer disease: modulations by plant-derived natural compounds and dietary interventions. Biomolecules. (2019) 9:289. doi: 10.3390/biom907 0289

64. Magzoub MM, Prunello M, Brennan K, Gevaert O. The impact of DNA methylation on the cancer proteome. PLoS Comput Biol. (2019) 15:e1007245. doi: 10.1371/journal.pcbi.100 7245

65. O'Rourke CJ, Lafuente-Barquero J, Andersen JB. Epigenome remodeling in cholangiocarcinoma. Trends Cancer. (2019) 5:335-50. doi: 10.1016/j.trecan.2019.05.002

66. Jusakul A, Cutcutache I, Yong CH, Lim JQ, Huang MN, Padmanabhan $\mathrm{N}$, et al. Whole-genome and epigenomic landscapes of etiologically distinct subtypes of cholangiocarcinoma. Cancer Discov. (2017) 7:111635. doi: 10.1158/2159-8290.CD-17-0368

Conflict of Interest: The authors declare that the research was conducted in the absence of any commercial or financial relationships that could be construed as a potential conflict of interest.

Copyright (c) 2021 Du, Zhang, Yin, Wang, Yan, Xie, Zheng, Zheng, Li and Song. This is an open-access article distributed under the terms of the Creative Commons Attribution License (CC BY). The use, distribution or reproduction in other forums is permitted, provided the original author(s) and the copyright owner(s) are credited and that the original publication in this journal is cited, in accordance with accepted academic practice. No use, distribution or reproduction is permitted which does not comply with these terms. 